The authors assessed data from 193 randomized, controlled trials of pharmacologic $(n=119)$ or nonpharmacologic $(n=74)$ treatments for rheumatoid arthritis or osteoarthritis of the hip or knee. Analysis was limited to primary reports from full-text papers published over a 5 -year period. Trials that included both pharmacologic and nonpharmacologic treatments, or both medical conditions, were excluded.

Studies of pharmacologic treatments reported data on harm more often than nonpharmacologic studies $(86.5 \%$ versus $47.3 \%)$. Data on treatment withdrawal due to adverse events, and on the nature, frequency and severity of adverse events, were more frequently reported and more detailed in pharmacologic treatment studies $(P<0.001$ for all). This relationship persisted after adjustment for potential confounding factors.

Strict regulatory requirements exist for reporting harm in pharmacologic treatment studies, but do not apply to nonpharmacologic treatments other than technical devices. Systematic collection of these data, according to CONSORT recommendations, would permit the detection of unexpected, delayed, or apparently unrelated adverse events in nonpharmacologic treatment studies. Funding is, however, an issue: drug manufacturers must report data on harm and therefore will fund the necessary studies. For nonpharmacologic treatments, harm might only be reported anecdotally in case reports.

\section{Caroline Barranco}

Original article Ethgen $\mathrm{M}$ et al. (2005) Reporting of harm in randomized, controlled trials of nonpharmacologic treatment for rheumatic disease. Ann Intern Med 143: 20-25

\section{PDC-derived interferon- $\alpha$ is a master cytokine in psoriasis development}

Researchers have discovered a key innate immunity pathway for triggering psoriasis: interferon- $\alpha$ (IFN- $\alpha$ ) derived from plasmacytoid predendritic cells (PDCs) activates and expands pathogenic $T$ cells, causing development of skin lesions.

The investigators hypothesized that IFN- $\alpha$ produced by PDCs might contribute to the pathogenesis of psoriasis, supported by previous observations. To test this hypothesis, Nestle et al. first assessed the distribution of PDCs. They found that in normal controls there was no PDC accumulation in skin, but PDCs accumulated in plaque lesions, and surprisingly, in nearby uninvolved skin, in patients with psoriasis.

IFN- $\alpha$ mRNA levels in psoriatic lesions were the same as those in normal skin; however, psoriatic lesions showed increased expression of an IFN- $\alpha$-inducible gene and a marker of IFN- $\alpha$. This suggested that IFN- $\alpha$ had been produced earlier during the development of the psoriatic lesion, which was confirmed by tests in a mouse xenograft model of human psoriasis. To investigate the role of IFN- $\alpha$ in psoriasis, IFN- $\alpha$ and IFN- $\beta$ signaling were blocked, resulting in complete inhibition of activation and expansion of pathogenic $T$ cells. Immunostaining showed PDCs were the principal IFN- $\alpha$-producing cell, and tests in mice showed PDC-derived IFN- $\alpha$ was necessary, and also sufficient, to drive psoriasis in prepsoriatic skin.

It was concluded that new strategies targeting PDCs and PDC-derived IFN- $\alpha$ should be considered for prevention and early therapeutic intervention in psoriasis.

Rachel Murphy

Original article Nestle FO et al. (2005) Plasmacytoid predendritic cells initiate psoriasis through interferon- $\alpha$ production. J Exp Med 202: 135-143

\section{Interleukin-17 inhibition suppresses flares of arthritis}

Blocking interleukin-17 (IL-17) prevents joint inflammation and bone erosion during reactivation of experimental arthritis, a murine study has found. Earlier studies have clearly implicated IL-17 in the development of arthritis, but the role of IL-17 in flare-up reactions of arthritis had not previously been elucidated.

In the study performed by Koenders et al., induction of antigen-induced arthritis (AIA) in male C57B1/6 mice was performed by immunization with $60 \mu \mathrm{g}$ of methylated bovine serum albumin. At 3 weeks of arthritis, a flare was induced by intra-articular injection of $2 \mu \mathrm{g}$ of bovine serum antigen into the arthritic joint. Measurements of IL-17 mRNA showed that following the primary AIA, IL-17 mRNA expression is upregulated, and that following antigen rechallenge, IL-17 mRNA expression is rapidly

\section{GLOSSARY}

CONSORT

The Consolidated

Standards of Reporting

Trials statement; an

extension has recently been

added on the reporting of

harm in randomized trials 